\title{
Un danseur illimité
}

À propos de Énora Rivière, Ob.scène. Récit fictif d'une vie de danseur, Pantin, Centre national de la danse, collection " Parcours d'artistes ", 2013, 128 pages

\section{Alice Gervais-Ragu}

\section{(2) OpenEdition}

\section{Journals}

Édition électronique

URL : http://journals.openedition.org/danse/723

DOI : $10.4000 /$ danse. 723

ISSN : 2275-2293

Éditeur

ACD - Association des Chercheurs en Danse

Référence électronique

Alice Gervais-Ragu, «Un danseur illimité », Recherches en danse [En ligne], Actualités de la recherche, mis en ligne le 25 avril 2014, consulté le 19 septembre 2020. URL : http://journals.openedition.org/ danse/723; DOI : https://doi.org/10.4000/danse.723

Ce document a été généré automatiquement le 19 septembre 2020.

association des Chercheurs en Danse 


\section{Un danseur illimité}

À propos de Énora Rivière, Ob.scène. Récit fictif d'une vie de danseur, Pantin, Centre national de la danse, collection « Parcours d'artistes », 2013, 128 pages

Alice Gervais-Ragu

\section{RÉFÉRENCE}

Énora Rivière, Ob.scène. Récit fictif d'une vie de danseur, Pantin, Centre national de la danse, collection « Parcours d'artistes », 2013, 128 pages

\section{De la multiplicité du danseur fictionné}


Ob.scène. Récit fictif d'une vie de danseur est un ouvrage d'Énora Rivière, paru en septembre 2013 aux éditions du Centre national de la danse, dans la collection Parcours d'artistes. Danseuse et chorégraphe, Énora Rivière mène parallèlement une activité de chercheuse en danse. Issue du CNR de Tours et de la formation ex.e.r.ce à Montpellier, elle obtient également un DEA au département danse de l'Université Paris 8. Elle travaille auprès de différents chorégraphes (Gilles Jobin, Mathilde Monnier, François Chaignaud, Cecilia Bengolea, Mickaël Phelippeau, Maud Le Pladec...) et plasticiens (Marie Reinert, Bill Durgin...). Conjointement, elle développe ses propres projets, relatifs notamment au point de vue du danseur-interprète depuis

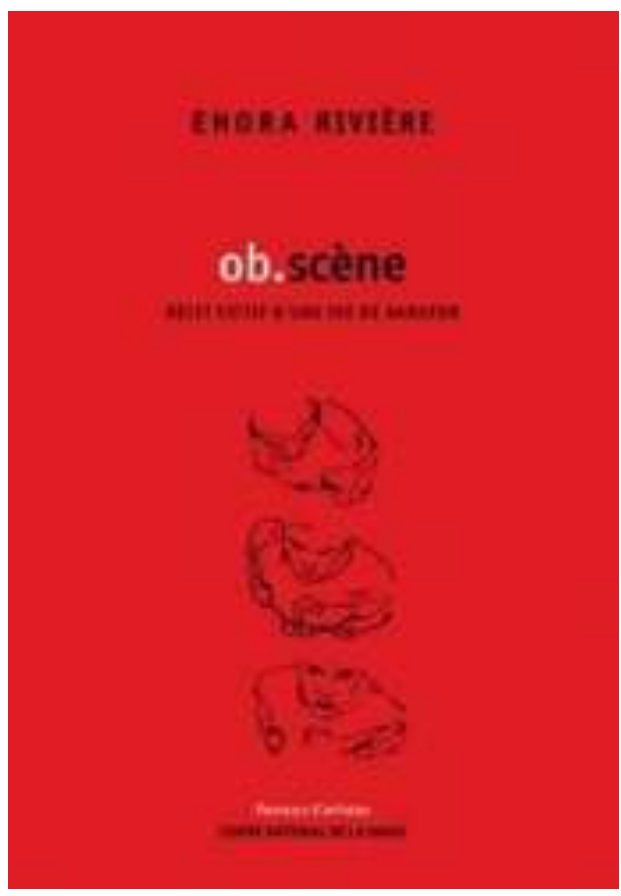
la pratique de son métier. Elle obtient ainsi l'Aide aux écritures chorégraphiques en 2006, et la Villa Médicis Hors les Murs en 2007, pour laquelle elle se rend à Rio, au Brésil. À partir d'entretiens menés avec des danseurs d'âges, de formations et de cultures différents, elle conçoit le livre Ob.scène, récit fictif d'une vie de danseur.

2 L'ouvrage d'Énora Rivière permet, à plusieurs titres, de renouveler les manières de se penser danseur - et/ou de le penser - aujourd'hui. Au prime abord, l'on observe l'objet acquis au Centre national de la danse, pour dix euros tout ronds : format court, couverture orange vif, titre à consonance vaguement subversive, petits paragraphes alternant noir et grisé, mise en page dynamique lui prêtent une apparence directe, presque incisive - le contenu du livre s'envisage alors naturellement à l'avenant. Mais Ob.scène, en réalité, s'avère plus complexe que cela: le texte fait bientôt mouche, construit comme un jeu s'articulant entre distance et proximité, au moyen de l'usage d'un tu et d'un je qui tour à tour intimise ou adresse le propos. L'air de rien, ob.scène s'impose bientôt, pour qui entend en découdre avec le danseur (et les fantasmes de représentation qui vont avec) comme un ouvrage majeur.

D'emblée, la première suggestion qu'Ob.scène fait au lecteur est de se laisser aller à une véritable expérience de vertige. En premier lieu de par la structure polyphonique du texte, qui s'articule autour de la répartition du je et du tu: se succédant les unes aux autres, les parties grisées prennent en charge le je, les parties noires sont dévolues au tu. Ici, le je s'origine en la perception du danseur dans son rapport intime à la scène, quand le tu extériorise le propos, telle la marque d'un dialogue intérieur adressé directement au lecteur. Ce je et ce $t u$, fonctionnant à rebours de personnages qui seraient prédéfinis par l'auteur, fabriquent ici deux instances, lesquelles commandent à la fois le flux et le fil du texte. En effet, on identifie à la fois : d'une part un personnage fictif multiple, qui prend en charge la mimésis du texte, au sens où il reproduit et restitue en les fictionnant des états vécus de danse ; et d'autre part un narrateur choral, lequel conduit la diégèse du texte, au sens où les actions mises en jeu et leur 
progression s'entrecroisent et/ou se superposent. Ces instances, à leur tour, travaillent alors à la fabrique d'un corps rêvé, un corps prolongé, sorte d'être de danse perçu audelà du tangible.

De l'axe méthodologique adopté pour la composition de ce je et de ce tu, et plus largement, de celle de l'ouvrage, l'on n'apprendra, en postface, que peu de choses : des entretiens menés par l'auteur avec "un groupe de danseurs de générations, de formations et de cultures différentes, [invité] à débattre, collectivement en studio pendant trente jours, à raisons de huit heures par jour, entre mars 2010 et janvier $2012 »$ (p. 125).

4 Toutefois, une fois le livre refermé, si d'aventure il arrive que l'on souhaite réinterroger son principe méthodologique, il peut être intéressant de revenir à la page 7 :

«Je pose des questions et tu y réponds. / Avec doute. Aisance. Nonchalance. / [...] Je pose des questions douteuses, l'air de rien, / grosses comme une maison. / Je ne pose pas une question. / J'en pose quatre. Six. Dix d'un coup. / Je ne parviens pas à poser la bonne question, / à détenir la bonne formulation. »

Relue a posteriori, cette introduction permet de mieux saisir la délicatesse de l'entreprise menée - et tout le soin mis à composer depuis le matériau brut. De ce dernier qu'on imagine dense, follement élastique, foisonnant, Énora Rivière conçoit une fiction kaléidoscopique, qui permet aux récits exploités de résonner tout à la fois avec la force du vécu et du fantasme. C'est dans l'oscillation constante du texte, entre diégèse et mimésis, qu'il faut rechercher cet effet d'élasticité. Cette particularité provoque une lecture déstabilisée, laquelle tend à se calquer sur le processus performatif de l'écriture elle-même : ainsi lecture et écriture se tiennent-elles par une puissance partagée du chancellement, créant une zone perceptive singulière où le personnage/narrateur - symbolisé par le je/tu - et le lecteur sont mutuellement engagés en une danse tangente. Ce phénomène augmente encore en ce que le lecteur entre tacitement en contrat d'empathie kinesthésique et sensorielle avec l'écriture, spécialement lors de certaines descriptions de vécus chorégraphiques:

"J'approche de ce point fixe où je vais décider de me poser. J'y suis. Mes jambes et mes pieds ne s'arrêtent pas. Le paysage continue de défiler dans ma tête. Devant moi, les couleurs, les odeurs, les bruits. Je continue de marcher sur ce point. Je commence à sentir mes bras qui balancent fort. Mon regard est projeté vers l'avant, ma pensée se concentre sur tout ce que j'ai exactement traversé, tout ce qui m'a envahi la tête, tout ce que j'ai imaginé et que j'ai arpenté pendant cinquante-sept minutes, les personnes que j'ai croisées, tout ça continue de défiler, toute cette mémoire dans les moindres détails. Je continue de marcher vers l'avant, mon but, c'est de marcher vers l'avant. » (p. 56).

Cet extrait permet d'entrevoir combien l'usage du temps présent, couplé à un sens accru du détail dans la description des faits, contribuent à produire une forte immédiateté perceptive chez le lecteur, qui devient alors lui-même acteur de ce qu'il lit. Pour aller plus loin, conduit par cette expérience de lecture participative, il peut aussi prolonger à loisir cette empathie en quittant la lecture, afin de mieux se connecter à ses propres sensations : il arrive ainsi que la lecture travaille et perdure dans le corps même du lecteur, le ressaisissant par exemple lorsqu'il se trouve en train de marcher, de travailler - ou de danser. 


\section{Poétique et sociologie d'une vie de danseur}

5 La trame du livre semble s'articuler sur une aire double, qui contiendrait à la fois un axe sociologique et une dimension poétique, sans que ceux-ci ne puissent réellement se distinguer dans le texte, les deux étant plus à penser en termes d'entrelacs que de scission à proprement parler. Cependant, si le livre esquisse bien une trame sociologique, c'est peut-être davantage dans l'a posteriori accompli par le geste de lecture qu'on l'identifie. Le projet du livre, s'apparentant avant tout à un travail de composition - à la fois littéraire et kinesthésique - ne procède pas, en effet, d'un pacte de facture sociologique. C'est donc le lecteur qui peut, potentiellement, y reconnaître des enjeux sociologiques, au sens où il est pris dans les problématiques posées par un milieu à une époque donnée. En particulier, cette double articulation permise par la structure du livre se cristallise lorsque le récit rend compte de la manière dont peut se construire un désir en danse :

" Au début, tu avais une perception très romantique de la question de l'engagement dans une pratique artistique. Ça t'a poussée dans des retranchements très intenses, de même la perspective d'une vie reliée à la création. Pour toi, c'était un espace d'extrémité, des partis pris que tu avais envie de défendre, l'endroit où tu avais envie d'être. [...] Ce romantisme, cette croyance et ce désir reviennent. Tu as envie de refaire des choses impossibles, de t'engager dans des projets improbables, de repartir dans cette forme de découverte et de conquête. » (pp. 35-36).

Cette approche couplée, depuis ses axes respectifs, renvoie en particulier à deux ouvrages: Je suis une école de Boris Charmatz et Danser. Enquête dans les coulisses d'une vocation de Pierre-Emmanuel Sorignet ${ }^{1}$.

6 Le premier en raison de son caractère de livre-performance, un livre qui serait presque dansé avant que d'être écrit, voire dansé en s'écrivant, un livre enfin où l'infini des possibles d'une vie de danseur pourrait s'épandre sans limite, s'autorisant à se rêver. Faisant suite au projet Bocal ${ }^{2}$, Je suis une école est lui aussi spéculé comme le récit ad libitum de se rêver/penser danseur aujourd'hui. À l'instar de Je suis une école, Ob.scène s'attèle à un véritable projet de déconstruction des représentations fantasmées du statut, de la vie et du métier de danseur, notamment dans son traitement de l'expérience de la scène et de ce qui s'y passe avant d'y entrer, pendant, puis après en être sorti. Débarrassé de toute indication définitive de genre - bien que le narrateur soit tour à tour féminisé ou masculinisé, au gré sans doute des supports initiaux de témoignages -, d'indication d'âge, d'identité, de nationalité ou d'appartenance géographique, le danseur fictionné d'Énora Rivière se livre de manière très intime, presque intéroceptive. Et de ce danseur, l'auteur parvient à recréer l'épaisseur, mais aussi la moiteur et la sueur:

« Ça fait dix minutes que j'ai envie de roter. J'attends avec impatience le moment où je vais pouvoir tourner le dos au public et roter. Je rote. Ma main est pleine de sueur. Ça glisse. Ma conscience se disperse, mon imaginaire entame un long voyage. La nausée, les étourdissements arrivent, j'ai l'impression que le support duquel je suis arrimé bouge, m'échappe. » (p. 32).

En cela également, persistent à la lecture d'Ob.scène les relents de Je suis une école, en particulier en son préambule: "Nous substituons une folie pédagogique à l'entraînement régulier du danseur - un allergène. Nos théories sentent, nos pratiques peuvent blesser, nos idées de cours sont des cours, nos cours sont nos performances, nos performances sont nos exercices quotidiens et glissants ${ }^{3}$ ». 
7 Ces temps-là d'Ob.scène, où l'expérience de la scène est décrite, sont donc symbolisés par une police grisée et par l'usage spécifique du je, lesquels procèdent à la fois d'une distinction formelle et d'un accès marqué au sensible. Très vite, l'alternance entre le je et le $t u$, entre les lettres noires et grises, confère à une atmosphère onirique ou à un processus cinématographique, provoquant des impressions de séquences d'actions pas finies ou déjà vues, que l'on perd puis que l'on retrouve.

8 À ce titre, le processus d'illimitation perceptive participe, d'une certaine façon, à construire une figure de danseur-monstre. En effet, le parti-pris d'une restitution empirique des différents états que peut traverser un danseur au cours de sa carrière va, peu à peu, donner lieu à une monstration presque spectaculaire des différents phénomènes en jeu. En particulier, l'on observe comment le choix du flot et du rythme du texte organise une parole très orale, où l'on peut presque entendre l'écho des entretiens d'origine. En cela, le verbe, en son débit, agit comme le flot d'un slam, qui à son tour ré-évoque le projet à l'œuvre dans Je suis une école: «Si je commence, cela ne s'arrêtera plus, le flux va trouver des pentes infinies, ce sera un barrage béant tout à coup, si j'ouvre mes doigts sur la chose, elle va grossir encore, je serai intarissable ${ }^{4} »$. Le choix du temps présent n'est évidemment pas étranger à créer cette atmosphère d'immédiateté, où souvent coïncident l'action et son anticipation, le faire et le vivre, spécialement lorsqu'il s'agit, dans le texte d'Énora Rivière, de restituer le vertige qui saisit le danseur en scène, en même temps qu'il se doit de le gérer afin d'effectuer son geste : «Je continue de marcher vers l'avant, mon but, c'est de marcher vers l'avant. STOP! Je lève les deux mains comme si on allait me fusiller. Je m'arrête. Je saute. Comme si c'était la dernière fois » (p. 56). Il est à noter qu'ici, le danseur a lui-même recours à un processus d'intégration de son geste presque cinématographique, dans le procédé dramaturgique qu'il met alors en place: le récit qu'il établit provoque instantanément chez lui une image mentale, laquelle à son tour vient irriguer sa danse.

Ob.scène peut par ailleurs rappeler certains des enjeux présents dans l'ouvrage de Pierre-Emmanuel Sorignet. D'une part, il s'avère que les deux auteurs sont l'un et l'autre engagés dans une pratique chorégraphique professionnelle, qui les a considérablement avantagés, si l'on peut dire, dans l'élaboration de leurs ouvrages respectifs. Mais les procédés d'implication, de recherche, de constitution et de traitement des matériaux diffèrent à plusieurs titres. Là où le premier menait une enquête participative sur une durée de plus de dix années, dans laquelle il choisissait d'anonymiser les participants au moyen de pseudonymes, la seconde imagine et fédère, durant quasiment deux ans, des ateliers regroupant une dizaine de danseurs - dont on peut lire les noms cités à la dernière page de l'ouvrage. L'objectif : « [...] Se remémorer et inventer à plusieurs. Échanger. Enregistrer. Transcrire. Écrire. Rendre collective une expérience singulière » (p. 125). L'anonymat des danseurs engagés dans l'expérience intervient ici à la fois partiellement - du fait des noms cités en dernière page - et à titre formel - par le jeu stylistique induit par le recours au je et au tu.

D'autre part, à l'instar de Danser. Enquête dans les coulisses d'une vocation, le livre d'Énora Rivière constitue lui aussi un document générationnel, miroir des préoccupations actuelles des danseurs. En premier lieu dans les formulations de ces derniers - où l'on observe, par exemple, une évolution dans les modalités de discours des danseurs, comme pour l'usage ringardisé du mot répétition auquel sont préférés, avec le temps, les termes projet ou création. En second lieu dans les intérêts et questionnements des danseurs contemporains: en particulier, l'attrait pour les pratiques somatiques, les 
remises en question des manières de se vivre danseur aujourd'hui, les rapports avec les chorégraphes, le désir de rébellion face au système institutionnel, ou encore la définition de l'artiste et de son idéal. Écrits à une époque d'extrême précarisation du métier de danseur, les deux ouvrages coïncident de facto dans le rapport préoccupant qu'ils font des réalités économiques avec lesquelles le danseur professionnel doit désormais composer. La question sensible de la reconversion est par ailleurs observée dans les deux ouvrages. En outre, les deux projets font état de danseurs de moins en moins « prêts à tout » dans leur implication avec les chorégraphes. Notamment, se pose la question du droit d'auteur ${ }^{5}$ et du danseur auteur de sa danse: à l'heure où les processus de création chorégraphique évoluent, où désormais la majorité des chorégraphes s'appuient sur les matériaux apportés par les danseurs, où enfin chorégraphes et danseurs font mutuellement retour sur le travail effectué, qui, in fine, serait auteur de quoi ${ }^{6}$ ? Outre l'implication dans l'écriture, Ob.scène pointe également la question de l'appropriation des œuvres par les danseurs, relativement aux multiples interprétations qu'ils en font. Ce qui pose d'une part, la question de l'accès à une légitimité d'auteur par la constance et la multiplicité de la pratique d'une œuvre, d'autre part celle de la perspective de la vitalité d'une œuvre par les interprétations qu'elle génère.

11 À cet égard, le propos d'Énora Rivière vient prolonger, mais aussi nuancer celui de Pierre-Emmanuel Sorignet, et amplifie ce qui demeurait en germe dans ce dernierl'on peut ici arguer d'un léger quoique déterminant écart générationnel entre les deux auteurs, mais aussi entre les deux corpus de danseurs sur lesquels s'appuient la matière de discours et de ressenti pour l'une, et le travail d'enquête pour l'autre : dans Ob.scène, on perçoit en effet davantage combien les pratiques en danse se sont modifiées au cours de ces deux dernières décennies. De l'époque où l'on faisait des pièces «sans discussion, conversation ni débat » (p. 92), où l'injonction «Fais ça » (p. 96) primait chez le chorégraphe sur la mise en débat éventuelle du danseur, on est passé à l'ère des échanges, où le danseur s'attèle désormais à être à l'écoute de ce qui se passe dans son corps. En outre, le silence de l'interprète n'est plus forcément paradigmatique de ce qui se passe en scène, au sens où l'utilisation de la parole sur scène vient, là encore, modifier considérablement pratiques et discours en danse. De la même manière, concernant l'évolution des méthodes de formation du danseur, le narrateur choral d' Ob.scène pose la question d'être « informé " plutôt que "formé ", voire remet en cause l'opportunité, en danse, de préférer "former des petits soldats" plutôt que "des chercheurs et des philosophes » (pp. 105-107).

\section{De la poétique politique}

12 De ce point de vue, la force d'Ob.scène constituerait aussi une expérience profondément subversive, dans la mesure où l'ouvrage non seulement construit la politisation d'une parole, mais encore rend compte dans le temps de cette construction et de ses modalités. Aussi, plus que d'un engagement politique à proprement parler, qui passerait par exemple par une syndicalisation massive de la société chorégraphique, ou par des prises de position publique pour telles ou telles causes, Ob.scène laisse entrevoir la possibilité d'une puissance politique par les corps : « Tu penses que faire de son corps son principal outil est beaucoup plus intéressant comme pratique de vie que de détenir une carte de syndicat» (p. 70). Le propos n'est pas sans résonner avec celui de Gérard 
Mayen : prenant appui sur un corpus constitué de cinq chorégraphes contemporains ${ }^{7}$, le critique interroge l'actuelle désaffection en matière de manifeste en danse :

«Ces jeunes personnalités sont aujourd'hui porteuses d'actes chorégraphiques remarqués. Or, le contexte où ils évoluent semble se passer très bien de déclaration collective manifeste. Cela alors que voici quinze ans, l'adresse d'une lettre, le 20 août 1997, à la ministre de la Culture d'alors (Catherine Trautmann), ouvrait cinq années d'intenses prises de position, qu'on retient comme le mouvement des Signataires du 20 août. Lorsque la conversation s'engage avec chacun des sus-cités, toujours est d'abord énoncée la portée politique en soi de leur geste chorégraphique. [...] le geste de danse se distinguerait-il, en tant que lui-même participant de l'élaboration d'une personnalité du langage ? Je bouge, donc je dis : autrement qu'en mots, je dis en direct aussi ${ }^{8} »$.

13 Le portrait de danseur dessiné dans le livre d'Énora Rivière est celui d'un danseur contemporain, autonome dans sa pensée comme dans son rapport au sensible. En cela, il apparaît comme un miroir assez fidèle de l'actuelle génération de danseurs, c'est-àdire de celle en place et active aujourd'hui, ne se laissant rien confisquer de sa capacité d'énonciation et/ou de prise de position. À ce titre, l'un des enjeux d'ob.scène, en ce qu'il fictionne d'abord une parole de danseurs.euses, semble répondre à cette interrogation posée par Gérard Mayen: s'il s'y dit que la force du politique serait implicitement portée par les corps, Ob.scène n'en demeure pas moins un livre, un écrit, que l'on peut, d'une certaine manière, apparenter à un manifeste. De page en page, opère en effet la puissance de la teneur poétique du texte, mêlée à une portée sociologique d'un genre nouveau, dont on repère in fine tout l'enjeu politique.

Pour le lecteur, l'intérêt d'Ob.scène est donc au moins double. D'une part, le livre porte en lui une promesse, qu'il tient, d'un voyage singulier du sensible, habilement mené par le biais de savants croisements perceptifs et narratifs. C'est ainsi l'occasion de faire l'expérience d'une plongée poétique à différents degrés, où le lecteur se laisse aller au gré du flux et/ou du fil du texte. L'une des forces d'ob.scène tient probablement aux choix d'appropriation du texte qu'il laisse faire au lecteur. Ainsi, le danseur illimité que construit Énora Rivière fait-il émerger la perspective d'un lecteur illimité dans la possibilité de ses choix d'incorporation perceptive. D'autre part, Ob.scène permet au lecteur, qu'il soit lui-même danseur ou non, de se saisir des enjeux intellectuels, mais aussi politiques d'une existence de danseur. En conclusion, l'on dégagera avant tout l'extraordinaire vitalité d'une pensée en marche, mais aussi du statut accordé à cette pensée, laquelle prend racine, rappelons-le, depuis l'expérience du danseur-interprète et non du chorégraphe. En cela, la démarche est rare, du moins suffisamment pour être remarquée. C'est pourquoi Ob.scène porte en son creux une ambition particulière, celle de statut de modèle du genre, potentiellement suivi par de futurs positionnements d'envergure chez les prochaines générations de danseurs-interprètes. À suivre, donc.

BIBLIOGRAPHIE

CHARMATZ Boris, Je suis une école, Paris, Les Prairies ordinaires, 2009. 
SORIGNET Pierre-Emmanuel, Danser. Enquête dans les coulisses d'une vocation, Paris, La découverte, 2010.

MAYEN Gérard, «Seuls ensemble. De la possibilité d'un manifeste en danse » in Mouvement, $\mathrm{n}^{\circ} 70$, juillet-octobre 2013, pp. 23-25.

\section{NOTES}

1. CHARMATZ Boris, Je suis une école, Paris, Les prairies ordinaires, 2009 ; SORIGNET PierreEmmanuel, Danser. Enquête dans les coulisses d'une vocation, Paris, La Découverte, 2010.

2. En 2003, le chorégraphe Boris Charmatz et l'Association Edna ont mis en place un dispositif de recherche et de création pédagogiques intitulé Bocal. Il s'agissait de mettre l'école à l'épreuve de l'esthétique et du politique en réunissant une quinzaine de personnes de 20 à 30 ans : danseurs, médecin, plasticiens, designers, écrivains, circassien et musiciens. Tous se sont engagés dans une aventure «sans fin » dont le but était de penser l'école comme puissance de création, d'activer son potentiel émancipateur. Ce projet, qui interrogeait l'institution sans pour autant chercher à fonder un nouveau modèle, a généré un vaste répertoire d'idées, de débats et de propositions, entre action et utopie, création et enseignement. Je suis une école donne ainsi à comprendre comment la recherche d'une pédagogie singulière aide à conquérir une autonomie. Source : http://www.lesprairiesordinaires.com

3. CHARMATZ Boris, Je suis une école, op. cit, p. 1.

4. Ibid., p. 3.

5. Sur cette question juridique, nous renvoyons à l'article d'Aurore Vinant dans le numéro 2 de Recherches en danse (ndlr).

6. Ces questions sont traitées par différents articles du numéro 2 de la revue Recherches en danse, notamment ceux de Sarah Chaumette ou Marie Quiblier (ndlr).

7. Marianne Baillot, Cecilia Bengolea, François Chaignaud, Kevin Jean, Marcela Santander.

8. «Seuls ensemble. De la possibilité d'un manifeste en danse" in Mouvement, $n^{\circ} 70$, juilletoctobre 2013.

\section{RÉSUMÉS}

L'ouvrage d'Énora Rivière propose une plongée singulière dans le vécu du danseur des années 2010, au moyen d'un processus fictionnel établi à partir de témoignages de danseurs-interprètes, recueillis puis traités par l'auteur. Le texte s'articule principalement autour d'un axe à vocation poétique et sensible, mais fait également émerger une dimension sociologique, dont on se saisit comme d'un authentique outil de savoir, dans la perspective d'appréhender au plus intime la scène chorégraphique française du point de vue de l'interprète. Fort de ces deux approches croisées a priori opposées, Ob.scène construit autant qu'il produit une expérience de lecture tout à fait inédite, où empathie sensorielle et kinesthésique vient se mêler à une expérience de regard critique sur le milieu de la danse.

Énora Rivière's work offers a singular plunge into the life and experiences of dancers today. It is a poetic work of fiction based on the true confessions of dancers gathered by the author. There is a 
social dimension that can be used as a great tool for grasping the different, and furthermore, very personnal realities at work in the french choreographic scene. This double approach, both documentary and narrative creates a reading experience where we move, and are moved between identification and truly critical positions.

\section{INDEX}

Keywords : dancer, biography, fiction, politics

Mots-clés : danseur, biographie, fiction, politique

\section{AUTEURS}

\section{ALICE GERVAIS-RAGU}

Danseuse et chercheuse, Alice Gervais-Ragu travaille et participe à divers projets : notamment, elle crée dans les années 2000 la compagnie Trèsessis pour laquelle elle conçoit différentes pièces chorégraphiques, écrit et performe pour le groupe Séraphin Trap-Trap, danse auprès d'ensembles de musique contemporaine (Kunstellaire / New Flore), et participe à de multiples performances et interventions dansées (Entrée libre, Hors-Lits, One shot icaunais). En 2010 elle entame un cursus de recherche auprès du département danse de l'université Paris 8 , où elle obtient un Master 2 en 2012. Ses recherches portent sur les questions de genre et de survie en danse. Elle travaille actuellement à un projet de thèse de doctorat. 